\title{
First-Principles Description of Correlation Effects in Layered Materials
}

\author{
Andrea Marini, ${ }^{1}$ P. García-González, ${ }^{2}$ and Angel Rubio ${ }^{3,4}$ \\ ${ }^{1}$ CNR-INFM and European Theoretical Spectroscopy Facility (ETSF), Dipartimento di Fisica dell'Universitá di Roma "Tor Vergata", \\ Via della Ricerca Scientifica, I-00133 Roma, Italy \\ ${ }^{2}$ Departamento de Física Fundamental, Universidad Nacional de Educación a Distancia, Apartado 60.141, E-28080 Madrid, Spain \\ ${ }^{3}$ Institut für Theoretische Physik, Freie Universität Berlin, Arnimallee 14, D-14195 Berlin, Germany \\ ${ }^{4}$ Departamento de Física de Materiales, Facultad de Químicas Universidad del País Vasco, \\ European Theoretical Spectroscopy Facility (ETSF), Centro Mixto CSIC-UPV, Donostia International Physics Center (DIPC),
} E-20018 Donostia-San Sebastián, Spain

(Received 25 August 2005; published 5 April 2006)

\begin{abstract}
We present a first-principles description of anisotropic materials characterized by having both weak (dispersionlike) and strong covalent bonds, based on the adiabatic-connection fluctuation-dissipation theorem with density functional theory. For hexagonal boron nitride the in-plane and out-of-plane bonding as well as vibrational dynamics are well described both at equilibrium and when the layers are pulled apart. Bonding in covalent and ionic solids is also described. The formalism allows us to ping down the deficiencies of common exchange-correlation functionals and provides insight toward the inclusion of dispersion interactions into the correlation functional.
\end{abstract}

DOI: 10.1103/PhysRevLett.96.136404

Soft matter, biomolecules, and layered materials are examples of weakly bonded compounds, which constitute stringent tests for $a b$ initio electronic structure calculations. A successful description of such systems requires an accurate treatment of a whole range of interactions, from short-range covalent to long-range van der Waals (vdW) forces. Moreover, their study provides valuable hints about how to devise new approximations that describe at a reasonable computational cost very different bonding regimes. In this realm, layered materials represent a unique case. The Kohn-Sham (KS) method under the simple local density approximation (LDA) [1] is known to give reasonable structural properties near the equilibrium interlayer distance of systems like graphite [2] and hexagonal boron nitride ( $h$-BN) $[3,4]$. Surprisingly, the more elaborate exchange-correlation (XC) energy functional based on the generalized-gradient approximation (GGA) [5], while providing a good in-plane bonding, fails dramatically in the description of the equilibrium interlayer distance in $h$-BN and graphite, leading to completely unphysical results $[6,7]$. The mechanism responsible for this poor performance is not specific to GGA but to all functionals based on exact exchange [8] plus some LDA correlation (EXXc) (as illustrated below).

It has been hypothesized that this failure is due to the lack of description of vdW forces, which are the manifestation of long-range correlation effects [7]. However, LDA, without accounting for $\mathrm{vdW}$ interactions, does bind the $h$-BN and graphite layers. More importantly, recently proposed approximate nonlocal prescriptions beyond LDA or GGA in the vdW density functional (vdW-DF) framework [9], while describing vdW forces, result in a large overestimation of the equilibrium layer-layer distance [7]. Thus, available XC functionals cannot describe correctly
PACS numbers: 71.15.Mb, 63.20. $-\mathrm{e}, 71.10 .-\mathrm{w}, 71.15 . \mathrm{Nc}$

a layered material at different geometrical configurations. Consequently, there is a basic need to understand this severe breakdown and how to define a stable, successful scheme to calculate such structural properties. This is the goal of the present Letter. We demonstrate that a fully microscopic, parameter-free approach based on the adiabaticconnection fluctuation-dissipation theorem (ACFDT) [10] correctly describes the mechanical properties of $h$-BN near and far from the equilibrium configuration. We show that ACDFT does bind the $h$-BN layers at an equilibrium distance in excellent agreement with the experiment, accounting by construction for vdW forces [11,12]. In contrast, while LDA overbinds $h$-BN, GGA, EXXc and, obviously, any other prescription with a local or semilocal correlation part fail in describing the layers bonding.

$h$-BN has a wealth of important applications for high resistance and inert nanodevices $[3,4,13]$. Furthermore, the $h$-BN layer-layer interaction is weaker than in graphite (the paradigmatic example of layered material), thus being a better scenario to assess and understand the performance of KS-based schemes. The ACFDT has been applied so far only to jellium systems [12], molecules [14], or compact solids [15]. However, it is starting to be considered a promising $a b$ initio method because (i) it provides a reliable first-principles description of XC effects at a lower numerical cost than quantum chemistry methods or statistical quantum Monte Carlo techniques; (ii) it is naturally built into the KS scheme as a way to construct advanced $\mathrm{XC}$ energy functionals depending on the electron density $n(\mathbf{r})$ and on the set of occupied $\left[\phi_{v}(\mathbf{r})\right]$ and empty $\left[\phi_{c}(\mathbf{r})\right]$ KS orbitals. Indeed, according to the ACFDT, the total XC energy comprises of the exact-exchange energy $E_{\mathrm{X}}=$ $-\frac{1}{2} \int d \mathbf{r} d \mathbf{r}^{\prime}\left|\sum_{v}^{\text {occ }} \phi_{v}^{*}(\mathbf{r}) \phi_{v}\left(\mathbf{r}^{\prime}\right)\right|^{2} /\left|\mathbf{r}-\mathbf{r}^{\prime}\right|$, plus the correlation energy given exactly by 


$$
E_{\mathrm{C}}=-\int_{0}^{+\infty} \frac{d u}{2 \pi} \int_{0}^{\lambda} d \lambda \operatorname{Tr}\left(\hat{w}\left[\hat{\chi}_{\lambda}(i u)-\hat{\chi}_{0}(i u)\right]\right),
$$

(we will use atomic units unless otherwise stated). $\operatorname{Tr}$ denotes the spacial trace, and $\hat{\chi}_{\lambda}(i u)$ is the density-density response function at imaginary frequencies of a fictitious electron system interacting through a scaled Coulomb potential $\lambda w(\mathbf{r})=\lambda / r$ whose ground-state density equals the actual one. Thus, $\hat{\chi}_{0}(i u)$ is the noninteracting response function of this fictitious KS system:

$$
\chi_{0}\left(\mathbf{r}, \mathbf{r}^{\prime} ; i u\right)=2 \Re \sum_{v, c} \frac{\phi_{v}^{*}(\mathbf{r}) \phi_{v}\left(\mathbf{r}^{\prime}\right) \phi_{c}^{*}\left(\mathbf{r}^{\prime}\right) \phi_{c}(\mathbf{r})}{i u+\left(\varepsilon_{v}-\varepsilon_{c}\right)},
$$

where $\varepsilon_{v}\left(\varepsilon_{c}\right)$ are the occupied (empty) KS eigenenergies. The interacting response $\hat{\chi}$ can be obtained in the framework of time-dependent density functional theory $[16,17]$ by solving a Dyson-like equation:

$$
\hat{\chi}_{\lambda}(i u)=\left(\hat{\imath}-\hat{\chi}_{0}(i u)\left[\lambda \hat{w}+\hat{f}_{\mathrm{XC}, \lambda}(i u)\right]\right) \hat{\chi}_{\lambda}(i u),
$$

where $\hat{f}_{\mathrm{XC}, \lambda}(i u)$ is the XC kernel of the fictitious system with the scaled interaction $\lambda \hat{w}$. The random phase approximation (RPA) to $\hat{\chi}$ is obtained by setting $\hat{f}_{\mathrm{XC}}=0$. Note that, in contrast to other procedures put forward in the literature $[7,8]$, this scheme provides a correlation functional built at the same level than exact exchange.

The XC effects introduced by $\hat{f}_{\mathrm{XC}}$ on $\hat{\chi}$ for the calculation of $E_{\mathrm{C}}$ are considerably weaker than for the description of the optical properties of $h$-BN [18], as $\hat{\chi}(i u)$ is a very smooth function on the imaginary axis [19]. Therefore, we set $\hat{f}_{\mathrm{XC}, \lambda}(i u)=0$ in Eq. (3) and correct the wellknown deficiency of the RPA total correlation energy [21] via a LDA-like correction term (a prescription often labeled as RPA+) [20]. This scheme will be denoted in what follows as EXX-RPA + . Moreover, instead of solving self-consistently the KS equations for the EXX-RPA+ potential [22], we evaluated the XC energy using the KSLDA electronic structure as commonly done in ab initio many-body calculations [17]. We have explicitly checked that the use of GGA or EXXc KS states as a basis for the EXX-RPA+ expressions has little effect (less than 2\%) on the final XC energy.

Some technical details are relevant. The KS response function is computed in reciprocal space using well converged KS orbitals and eigenenergies obtained from the ABINIT code [23]. The interacting responses $\hat{\chi}_{\lambda}$ are then obtained for each vector in the Brillouin zone, imaginary frequency $u$, and coupling parameter $\lambda$ using the SELF code [24]. Finally, the RPA correlation energy is calculated according to Eq. (1) using a sixth order Gauss-Legendre sampling for $\lambda$ integration. The imaginary frequency integrals are done using two Gauss-Legendre grids, each typically comprising 24 points. This allows a well converged evaluation of the contributions from both small and large imaginary frequencies. The convergence with respect to the Brillouin zone sampling has been also carefully checked (the exchange energy is very sensitive to this sampling). Nonetheless, the critical convergence parameters from a computational point of view are the number of unoccupied bands and reciprocal space vectors in Eq. (2). The use of a plane-wave representation allows a systematic simultaneous convergence with respect to these two parameters. Further technical details, together with more applications to metallic systems and graphite, will be presented elsewhere.

Before studying the weak interlayer interaction in $h$-BN, it is illustrative to analyze the impact of the exchangecorrelation effects at the EXX-RPA+ level for two prototype compact structures: bulk Si (as covalent semiconductor) and $\mathrm{NaCl}$ (as ionic material). It is known that the standard XC functionals, like GGA, provide a good description of those systems. This is confirmed in the present ACFDT scheme as the impact in the lattice constant of the EXX-RPA + XC energy correction is below 0.5\%. However, the hybrid EXXc overcorrects the GGA results specially in the low density regime of bulk Si due to the LDA overestimation of the correlation energy for low densities (see inset of Fig. 1). Nevertheless, this does not compromise very much the accuracy of the EXXc approach since in this case the exchange energy dominates $\left[E_{\mathrm{X}}(11)-\right.$ $\left.E_{\mathrm{X}}(9.6) \approx 0.2 \mathrm{Ha} \gg E_{\mathrm{C}}(11)-E_{\mathrm{C}}(9.6) \approx 0.01 \mathrm{Ha}\right][25]$.

The previous scenario, where the variations of the exchange energy is at least 1 order of magnitude larger than correlation, changes completely in the case of $h$-BN. As displayed in Fig. 1, the variations of $E_{\mathrm{X}}$ and $E_{\mathrm{C}}$ around the equilibrium interlayer distance $d_{\mathrm{eq}}$ in $h$-BN are of the same order of magnitude (few $\mathrm{mHa}$ ) and at least 1 order of magnitude smaller than for $\mathrm{Si}$ or $\mathrm{NaCl}$. Thus, small errors

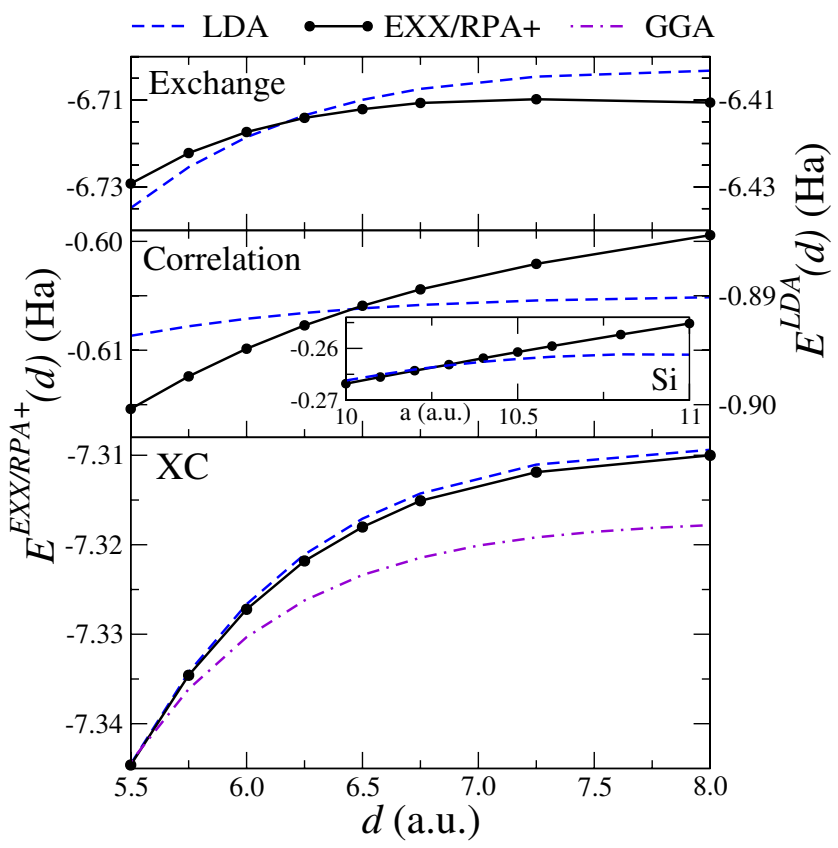

FIG. 1 (color online). The LDA, EXX-RPA +, and GGA exchange and correlation energies per unit cell for $h-\mathrm{BN}$ as a function of the layer-layer distance $d$ (the correlation energy of $\mathrm{Si}$ as a function of the lattice constant $a$ is shown in the inset). 
are more clearly visible and correlation plays a much more important role than in the case of the compact structures. Hence, a consistent description of exchange and correlation is needed to reproduce the delicate energetic interplay in the weak interlayer bonding of $h$-BN. This consistency is absent in the EXXc prescription and, as a consequence, it provides too large (or even unbound) interlayer equilibrium distances, as showed in Fig. 2. To a lesser extent, this problem is also present in the vdW-DF scheme designed to treat $\mathrm{vdW}$ forces in an approximate manner [7] that largely overestimates the equilibrium distance (6.86 compared to 6.24 a.u.), while underestimating the bulk modulus. The GGA follows the very same trend as the EXXc. This puts in evidence the fact that GGA exchange greatly improves upon the LDA (and, hence, provides better chemical properties of molecules), but does not do such a good job for correlation. Instead EXX-RPA+, which consistently treats exchange and correlation, correctly predicts $h$-BN to be stable, with structural parameters in excellent agreement with experiment (see Fig. 2). Furthermore, in contrast to LDA, GGA, and EXXc, EXX-RPA+ naturally embodies the correct long-range $d^{-4} \mathrm{vdW}$ power law dependence of the energy [6] as a function of interlayer distance, thus merging continuously the covalent and $\mathrm{vdW}$ regimes. This is confirmed in Fig. 2 where, for $d$ larger than 7.5 a.u., we observe that LDA deviates from the correct total energy dependence (given by EXX-RPA+). By looking at the LDA XC contributions in Fig. 1, we see that even if both $E_{\mathrm{X}}$ and $E_{\mathrm{C}}$ are poorly described, the XC energy dependence on the interlayer distance is similar to the ACDFT result. Nevertheless, the slope of the LDA XC energy is

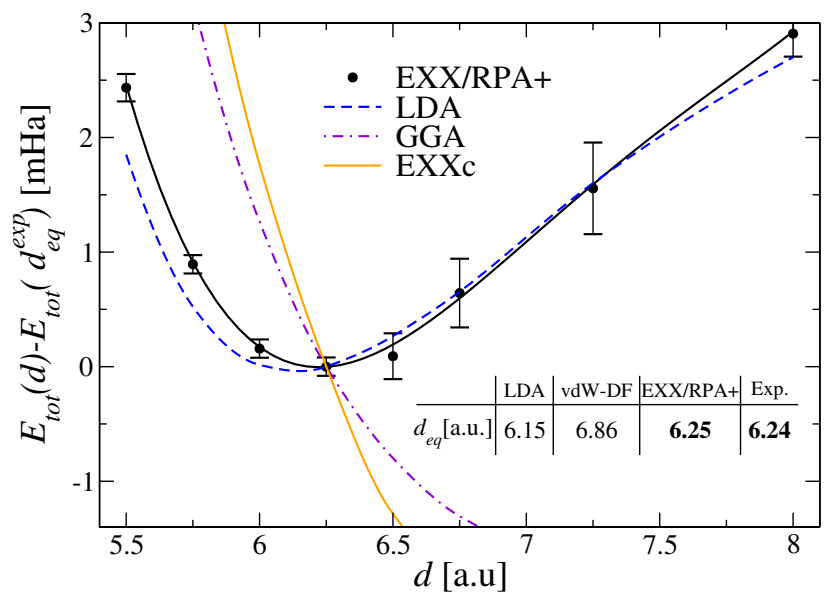

FIG. 2 (color online). Variation of the total energy per unit cell of $h$-BN versus the layer-layer distance $d$. While EXX-RPA+ binds the $h$-BN layers, GGA and EXXc dramatically fail. EXX-RPA + improves the equilibrium interlayer distance compared to vdW-DF [7] and LDA, whose good performance is merely due to the cancellation of errors. The EXX-RPA+ error bars come from the extrapolation technique used to extract the correlation energy from values corresponding to finite number of bands and reciprocal space calculations. The experimental equilibrium distance is taken from Ref. [26]. larger than the ACDFT one. This difference is responsible for the smaller interplane distance in the LDA as compared to EXX-RPA + , being the latter (6.25 a.u.) in stringent agreement with the experimental value $d_{\mathrm{eq}}^{\mathrm{exp}}=6.24$ a.u. [26]. Moreover, in the ACFDT the LDA bulk modulus B is increased by about $5 \%$ in better agreement with the experimental value (in contrast to the vdW-DF scheme [7] where $B$ is reduced). Therefore, while the relative success of the LDA around the equilibrium configuration is merely due to a cancellation of errors the EXX-RPA+ scheme provides better structural properties describing equally well the correlation and the exchange energy. This shows that the ACFDT ab initio scheme is suitable to study very different regimes, like $\mathrm{Si}$ and $\mathrm{NaCl}$, where the exchange energy dominates, or like $h-\mathrm{BN}$, where the success is bound to a correct balance of correlation and exchange contributions.

An additional severe test of the accuracy of the EXX$\mathrm{RPA}+$ method is given by the calculation of the vibrational spectra of $h$-BN. LDA and GGA in-plane lattice dynamics [3] reproduces fairly well the available experimental results. Similarly, the results of a frozen-phonon calculation within the present EXX-RPA+ scheme give in-plane modes at the high-symmetry points within a few percent from experiments (and LDA or GGA). The situation changes considerably for interplane modes where the LDA and GGA phonons differ by up to $30 \%$ when calculated at the experimental interplane distance (this is the only way to compare the results as GGA does not bind $h$-BN and LDA overbinds). Indeed, modes related to interlayer vibrations would be very much sensitive to errors in the correlation functional (as the typical variations in the correlation energy that has to be resolved are up to 2 orders of magnitude smaller for interlayer phonons than for structural characterization). Furthermore, recent second-order Raman experiments [27] have suggested that the interplane modes are greatly underestimated in the theoretical calculations. In particular, they quote a frequency value for the $\Gamma_{4}^{+}$phonon mode (where two $\mathrm{BN}$ planes rigidly slide against each other) of $310 \mathrm{~cm}^{-1}$, which is about a factor of 3 larger than the LDA $\left(90 \mathrm{~cm}^{-1}\right)$ or GGA $\left(122 \mathrm{~cm}^{-1}\right)$ values [28], computed at the experimental lattice parameters. If the experimental analysis based on overtones were correct it would imply that correlation effects (dispersion forces) are very important for those vibrations. This point can be directly addressed by the present EXX-RPA + scheme as we have shown that it describes properly the structural properties of $h$-BN and accounts at the same time by construction for the long-range vdW forces. Surprisingly, the calculated EXX$\mathrm{RPA}+\Gamma_{4}^{+}$phonon mode energy is $130 \pm 10 \mathrm{~cm}^{-1}$, regardless of the use of the theoretical or experimental lattice parameters. Therefore, it is clear that improving the description of XC effects upon LDA brings the $\Gamma_{4}^{+}$stiffer, still not close to the experimental assignment. However, this huge discrepancy can be drastically reduced if in the analysis of the experimental data we allow for the whole 
set of double-phonon processes (sum and difference) consistent with the selection rules of Raman scattering instead of considering only phonon overtones. If we do so, the new frequency assignment for the $\Gamma_{4}^{+}$phonon mode is about $125 \mathrm{~cm}^{-1}$ [29], still in disagreement with the LDA value but very close to the EXX-RPA+ result and to the GGA value (in spite of the fact that it does not bind $h$-BN). Therefore, EXX-RPA+ solves unambiguously this puzzling situation, providing an accurate method to evaluate the role played by the exchange-correlation effects.

In summary, we have shown that EXX-RPA+ correctly describes the structural properties, the on-plane and the out-of-plane phonon modes of $h$-BN. While both GGA and EXXc do not predict the $h$-BN layers bonding and LDA underestimates both the interlayer phonon mode frequency and interlayer distance, EXX-RPA+ provides a consistent, accurate and successful alternative scheme. Thus, we have shed light about the performance of standard ab initio techniques when applied to layered systems where any improvements upon LDA require equally accurate treatments of exchange and correlation effects. The EXX-RPA+ scheme presented here opens the field to more elaborate $a b$ initio applications in the wide field of sparse systems and soft matter.

The authors were partially supported by the EC Network of Excellence NANOQUANTA (NMP4-CT-2004-500198) and Spanish MCyT (FIS2004-05035-C03-03). P.G.G. thanks the Ramon y Cajal Program and A.R. the Humboldt Foundation. We benefited from discussions with J. F. Dobson, J. Jung, R. W. Godby, M. Grüning, J. Serrano, and L. Wirtz.

[1] W. Kohn and L. J. Sham, Phys. Rev. 140, A1133 (1965).

[2] J. C. Charlier, X. Gonze, and J.P. Michenaud, Europhys. Lett. 28, 403 (1994).

[3] G. Kern, G. Kresse, and J. Hafner, Phys. Rev. B 59, 8551 (1999).

[4] W. J. Yu, W. M. Lau, S.P. Chan, Z.F. Liu, and Q. Q. Zheng, Phys. Rev. B 67, 014108 (2003).

[5] J. P. Perdew, K. Burke, and M. Ernzerhof, Phys. Rev. Lett. 77, 3865 (1996).

[6] J. F. Dobson, K. McLennan, A. Rubio, J. Wang, T. Gould, H. M. Le, and B.P. Dinte, Australian J. Chem. 54, 513 (2001); J. F. Dobson, A. White, and A. Rubio, Phys. Rev. Lett. 96, 073201 (2006).

[7] H. Rydberg, M. Dion, N. Jacobson, E. Schröder, P. Hyldgaard, S. I. Simak, D. C. Langreth, and B. I. Lundqvist, Phys. Rev. Lett. 91, 126402 (2003).

[8] M. Städele, J. A. Majewski, P. Vogl, and A. Görling, Phys. Rev. Lett. 79, 2089 (1997).

[9] H. Rydberg, B. I. Lundqvist, D. C. Langreth, and M. Dion, Phys. Rev. B 62, 6997 (2000).

[10] D. C. Langreth and J. P. Perdew, Solid State Commun. 17, 1425 (1975); Phys. Rev. B 15, 2884 (1977).

[11] E. Hult, Y. Andersson, and B. Lundqvist, Phys. Rev. Lett. 77, 2029 (1996); W. Kohn, Y. Meir, and D. E. Makarov, Phys. Rev. Lett. 80, 4153 (1998).
[12] J.F. Dobson and J. Wang, Phys. Rev. Lett. 82, 2123 (1999); Phys. Rev. B 62, 10038 (2000); J. M. Pitarke and J.P. Perdew, Phys. Rev. B 67, 045101 (2003); J. Jung, P. García-González, J.F. Dobson, and R. W. Godby, Phys. Rev. B 70, 205107 (2004).

[13] See, for instance, E. Rapport, Ann. Chim. (Paris) 10, 607 (1985); N. G. Chopra, R. J. Luyken, K. Cherrey, V. H. Crespi, M. L. Cohen, S. G. Louie, and A. Zettl, Science 269, 966 (1995); A. Rubio, J. L. Corkill, and M. L. Cohen, Phys. Rev. B 49, 5081 (1994).

[14] F. Furche and T. Voorhis, J. Chem. Phys. 122, 164106 (2005); M. Fuchs and X. Gonze, Phys. Rev. B 65, 235109 (2002); M. Fuchs, K. Burke, Y.-M. Niquet, and X. Gonze, Phys. Rev. Lett. 90, 189701 (2003); F. Aryasetiawan, T. Miyake, and K. Terakura, Phys. Rev. Lett. 88, 166401 (2002); 90, 189702 (2003).

[15] T. Miyake, F. Aryasetiawan, T. Kotani, M. van Schilfgaarde, M. Usuda, and K. Terakura, Phys. Rev. B 66, 245103 (2002).

[16] E. Runge and E. K. U. Gross, Phys. Rev. Lett. 52, 997 (1984).

[17] For a review, see G. Onida, L. Reining, and A. Rubio, Rev. Mod. Phys. 74, 601 (2002).

[18] L. Wirtz, A. Marini, M. Grüning, and A. Rubio, cond-mat/ 0508421.

[19] For structural properties (i.e., energy differences in isoelectronic systems), there is evidence indicating that $\hat{f}_{\mathrm{XC}}$ plays a minor role $[12,14,20]$.

[20] S. Kurth and J.P. Perdew, Phys. Rev. B 59, 10461 (1999).

[21] M. Lein, E. K. U. Gross, and J. P. Perdew, Phys. Rev. B 61, 13431 (2000). The effect induced by this LDA-like correction is to shift rigidly the RPA $E_{C}$.

[22] Y. M. Niquet, M. Fuchs, and X. Gonze, Phys. Rev. A 68, 032507 (2003); J. Chem. Phys. 118, 9504 (2003); Y. M. Niquet and X. Gonze, Phys. Rev. B 70, 245115 (2004).

[23] See X. Gonze, J.-M. Beuken, R. Caracas, F. Detraux, M. Fuchs, G.-M. Rignanese, L. Sindic, M. Verstraete, G. Zerah, F. Jollet, M. Torrent, A. Roy, M. Mikami, Ph. Ghosez, J.-Y. Raty and D. C. Allan, Comput. Mater. Sci. 25, 478 (2002).

[24] SELF, http://www.fisica.uniroma2.it/ self/

[25] As is well known, LDA underestimates (overestimates) the absolute value of the exchange (correlation) energy. Both errors compensate and the LDA and ACFDT XC energies are very similar for compact structures.

[26] W. Paszkowicz, J. B. Pelka, M. Knapp, T. Szyszko, and S. Podsiadlo, Appl. Phys. A: Mater. Sci. Process. 75, 431 (2002).

[27] S. Reich, A. C. Ferrari, R. Arenal, A. Loiseau, I. Bello, and J. Robertson, Phys. Rev. B 71, 205201 (2005).

[28] We should stress that if the LDA phonon calculation is performed at the theoretical lattice constant, the corresponding $\Gamma_{4}^{+}$phonon frequency is $120 \mathrm{~cm}^{-1}$, in rather good agreement with the new assignment of the experimental $\Gamma_{4}^{+}$phonon mode [29].

[29] L. Wirtz et al., (unpublished). It turns out that the peak in the spectra attributed to an overtone of the out-of-plane acoustical (ZA) phonon mode along the M-K direction (observed at $920 \mathrm{~cm}^{-1}$ in Ref. [27]) is actually a combination of the optical and acoustical $\left(\Gamma_{4}^{+}\right)$phonons. 